\title{
Use of intravenous antibiotics for the treatment of community-acquired pneumonia in the emergency department
}

\author{
Anita G Carrie' \\ Thomas J Marrie² \\ 'Faculties of Pharmacy and \\ Pharmaceutical Sciences, ${ }^{2}$ Medicine \\ and Dentistry, University of Alberta, \\ Edmonton, $A B$, Canada
}

\begin{abstract}
Study objective: To determine the extent of intravenous (IV) antibiotic use for communityacquired pneumonia (CAP) in emergency departments, the practice patterns in seven emergency departments serving the adult residents of one Canadian city were observed.

Methods: An observational study of nonhospitalized adults diagnosed with CAP in seven emergency departments was conducted between November 15, 2000, and November 19, 2002. Data related to antibiotic treatment of CAP administered in the emergency department and patient-specific characteristics potentially predictive of IV treatment were collected.

Results: A total of 3512 subjects were identified, of which $4.9 \%$ received treatment with IV antibiotics. Cefuroxime and levofloxacin were the most commonly used IV agents, while orally-treated subjects primarily received a macrolide or levofloxacin. The proportion of subjects receiving IV antibiotics differed significantly among the seven sites: $1.4 \%-10.6 \%$ $(p<0.0001)$. Logistic regression identified a number of independent predictors of receipt of IV antibiotics including risk class, temperature, respiratory rate, study year, presence of vomiting, prior antibiotic treatment, and personal care home residence. However, these predictors did not explain intersite differences.

Conclusion: Only a small proportion of patients $(4.9 \%)$ presenting to the emergency department with CAP received IV antibiotics. While patient demographics and severity indicators influenced the likelihood of receipt of IV antibiotics, considerable intersite variation existed, despite adjustment for such factors.
\end{abstract}

Keywords: pneumonia, ambulatory care, antiinfective agents, infections, intravenous

\section{Introduction}

Community-acquired pneumonia (CAP) is a common infection with a reported incidence of between 1 and 12 episodes per 1000 persons per year, resulting in considerable morbidity, mortality, and healthcare costs (Marrie 1998). Guidelines for the management of CAP are widely available (Bartlett et al 2000; Mandell et al 2000); however, variation between recommended and actual practice is frequently reported (Gleason et al 1997; Fantin et al 2001). Practice patterns previously studied have included choice of antibiotic, rates of hospitalization, and length of stay (Gilbert et al 1998; Menendez et al 2001; Battleman et al 2002; Jin et al 2003). Variations in the route of antibiotic treatment, especially among outpatients, have received little attention, despite the considerable cost implications of intravenous (IV) treatment.

Current Canadian treatment guidelines do not make specific recommendations regarding route of treatment, except for patients requiring admission to the intensive care unit (Mandell et al 2000). However, patients at low risk treated on an outpatient basis receive predominantly oral antibiotics, while the majority of higher risk hospitalized subjects initially receive intravenously administered antibiotics (Gilbert 
et al 1998). While oral treatment is the norm among nonhospitalized patients, there is some support for initial treatment with parenteral antibiotics in the emergency department (ED) followed by oral administration (Moran 2001). The extent of this practice is unknown. We examined the extent and predictors of initial receipt of IV-administered antibiotics for CAP in EDs in one Canadian city.

\section{Methods}

This observational descriptive study was conducted at seven EDs that were associated with six hospitals and one community-care center, and service the needs of approximately 860000 residents of one Canadian city and surrounding areas. The study was approved by the Health Research Ethics Board of the university with which both authors are affiliated.

Beginning November 15, 2000, a "pneumonia critical pathway" was implemented at all seven sites in the region (see below). Patients 17 years of age or older were enrolled into the pathway if they presented to one of the study hospitals and were clinically diagnosed with communityacquired pneumonia based on two or more of the following symptoms: fever, cough, dyspnea, pleuritic chest pain, crackles, and findings of consolidation on chest examination plus an opacity on chest radiography (as interpreted by the attending physician). Patients were excluded from the pathway if they had physician-diagnosed or suspected aspiration pneumonia, tuberculosis, cystic fibrosis, were immunosuppressed (eg, human immunodeficiency virus infection, use of greater than $10 \mathrm{mg}$ per day of prednisone for more than one month or other immunosuppressive agents, active treatment for cancer, history of organ transplantation), pregnant or nursing, or if they required direct admission to the intensive care unit.

Based on our previously published work (Marrie et al 2000), a validated "critical pathway" for the management of pneumonia was developed with the goal of improving the quality and efficiency of care. A multidisciplinary team consisting of infectious disease specialists, general internists, respirologists, emergentologists, family physicians, pharmacists, nurses, respiratory therapists, and dieticians developed and implemented the pathway at all regional hospitals. Patients were evaluated and the "pneumonia severity of illness" score was calculated and risk class assigned using the patient's age, sex, comorbid illnesses, physical findings, and laboratory results (Fine et al 1997). For patients who were to be discharged from the ED, antibiotic therapy in accordance with published guidelines was recommended (Mandell et al 2000). No recommendation was made regarding IV antibiotic therapy for those who were to be treated on an ambulatory basis. Indeed oral antibiotic therapy was recommended for inpatients unless there was vomiting or hypotension.

Triage is defined as the "initial clinical assessment of patients" and is a term most often applied to patients assessed in the ED. Triage staff sort and prioritize patients for urgency of care based on information obtained at the time of presentation. Different triage systems have been developed, and, in Canada, the Canadian Triage and Acuity Scale (CTAS) is the nationally recognized standard for triage. This 5 -point scoring system grades patients from resuscitation (1) to deferrable (5) and is widely employed.

Research nurses were responsible for reviewing admission and ED discharge data on a daily (Monday to Friday) basis to identify all potential admitted or discharged CAP cases. They performed retrospective data collection using standardized case report forms. Data collection included, time of presentation to the ED, antibiotic(s) administered, and route and time of administration. Patient variables of interest included demographic data (eg, age, gender, personal care home residence), physical status (eg, presence of nausea or vomiting), physiologic data (eg, temperature, respiratory rate), previous treatment (eg, repeat visit for the same indication, antibiotic treatment in previous seven days), and triage score. In addition, risk class was assigned as described above. Data collection began November 15, 2000, and ended November 19, 2002.

\section{Statistical methods}

Univariate differences between IV and non-IV treated outpatients were assessed using Pearson chi-square and Wilcoxon rank sum tests as appropriate. Logistic regression was used to model the effects of site of care and patient characteristics on the probability of receipt of IV antibiotics. All potential explanatory variables were entered into the logistic model regardless of their statistical significance in univariate testing. Due to low numbers of subjects with triage scores of 1 or 5 , levels of this severity-related variable were collapsed and reclassified as "high" (triage score $=1$ or 2), "moderate" (triage score $=3$ ), and "low" (triage score $=4$ or 5). Subjects with missing triage scores were assigned the median based on treatment assignment (median $=3$ for both IV and non-IV treated patients). Subjects with missing time of presentation were assumed to have presented during the day. Refinement of the model was accomplished by stepwise removal of the least significant contributor, with minimum 
Table I Initial treatment of subjects with community-acquired pneumonia (CAP) presenting to seven emergency departments

\begin{tabular}{|c|c|c|c|c|c|c|c|}
\hline \multirow[b]{2}{*}{ Site } & \multirow{2}{*}{$\begin{array}{r}\text { All CAP } \\
\text { patients } \\
\mathbf{N}\end{array}$} & \multicolumn{2}{|c|}{$\begin{array}{c}\text { CAP patients } \\
\text { admitted to hospital }\end{array}$} & \multicolumn{2}{|c|}{$\begin{array}{c}\text { CAP patients } \\
\text { treated as outpatients }\end{array}$} & \multicolumn{2}{|c|}{$\begin{array}{l}\text { Proportion of outpatients } \\
\text { receiving IV antibiotics }\end{array}$} \\
\hline & & $\mathbf{N}$ & $\%$ & $\mathbf{N}$ & $\%$ & $\mathbf{N}$ & $\%^{\mathrm{a}}$ \\
\hline A & 669 & 124 & 18.5 & 545 & 81.5 & 58 & 10.6 \\
\hline B & 1769 & 985 & 55.7 & 784 & 44.3 & 57 & 7.3 \\
\hline C & 1003 & 590 & 58.8 & 413 & 41.2 & 20 & 4.8 \\
\hline D & 828 & 328 & 39.6 & 500 & 60.4 & 14 & 2.8 \\
\hline$E$ & 460 & 153 & 33.3 & 307 & 66.7 & 5 & 1.6 \\
\hline $\mathrm{F}$ & 871 & 393 & 45.1 & 478 & 54.9 & 12 & 2.5 \\
\hline G & 915 & 430 & 47.0 & 485 & 53.0 & 7 & 1.4 \\
\hline Total & 6515 & 3003 & 46.1 & 3512 & 53.9 & 173 & 4.9 \\
\hline
\end{tabular}

${ }^{a}$ Percentage is of those treated as outpatients.

Abbreviations: CAP, community-acquired pneumonia; IV, intravenous.

significance at the 0.05 level. The reduced, or parsimonious model, is reported with the effect of explanatory variables on the probability of receipt of an intravenous antibiotic reported as odds ratios with associated $95 \%$ confidence intervals. Statistical analysis was performed using Statistical Analysis System software version 8.2 (SAS Institute Inc, Cary, NC, USA).

\section{Results}

A total of 6515 potentially eligible subjects presented to the seven EDs, of which 3003 (46.1\%) were initially admitted and $3512(53.9 \%)$ were treated as outpatients (Table 1). Further, among outpatients, 173 (4.9\%) received treatment with IV antibiotics, 1523 (43.4\%) received oral antibiotic treatment, and $1816(51.7 \%)$ received no antibiotic treatment during the ED stay. Among IV-treated subjects, $59.0 \%$ received a single antibiotic, $39.3 \%$ received two antibiotic agents, and $2.0 \%$ received three or more different antibiotics. Among IV-treated subjects receiving a single antibiotic, levofloxacin (44.1\%) and cefuroxime (41.2\%) were most commonly administered. For those IV-treated subjects receiving two antibiotics during the ED stay, cefuroxime plus a macrolide (erythromycin, clarithromycin, or azithromycin) (72.1\%) and cefuroxime plus levofloxacin $(10.3 \%)$ combinations were most commonly observed. Greater than $99 \%$ of subjects receiving oral antibiotics were treated with a single agent, of which macrolides (erythromycin, clarithromycin, or azithromycin) (57.6\%) and levofloxacin $(39.7 \%)$ were the most commonly prescribed. Median time to discharge from the ED varied significantly between treatments; no treatment (2.9 hours), oral antibiotic (4.1 hours), and IV antibiotic (6.0 hours) $(p<0.0001)$. For subjects receiving antibiotic treatment there was no significant difference in the time to receipt of antibiotic agent; oral (3.2 hours), IV (3.4 hours) $(p=0.76)$.
The proportion of subjects receiving IV antibiotics varied significantly with risk class; I (2.9\%), II (4.7\%), III (5.6\%), IV $(7.3 \%)$, V $(17.2 \%)(p<0.0001)$. Subjects receiving IV antibiotics were significantly more likely to reside in personal care homes, have a recent history of vomiting, and have higher severity of illness, as indicated by triage score (Table 2). Differences in vital signs (eg, heart rate, respiratory rate) were also evident between IV and non-IV treated patients. Time of day of presentation to the ED had no effect on the likelihood of receipt of IV antibiotics.

Among outpatients, the proportion of subjects receiving IV antibiotics varied among sites from $1.4 \%$ to $10.6 \%$ (Table 1). The site with the highest proportion of IV antibiotic use (site A) was a "free-standing" ED not housed within an acute care hospital. There was considerable overlap in the physicians staffing this site and those staffing the ED with the second highest use of IV antibiotics (site B). A total of $506(92.8 \%)$ of the 545 outpatients presenting to site A and 388 (49.5\%) of 784 outpatients presenting to site B were treated by physicians staffing both sites. In an examination of only those 894 subjects treated by the overlapping physicians significant differences in receipt of IV antibiotics remained between the two sites; site A (11.1\%) and site B $(7.2 \%)(p=0.05)$. Overall, the likelihood of receipt of IV antibiotics decreased during the study period from $6.9 \%$ in year one to $3.0 \%$ in year two, and decreases were observed at all seven sites.

The multivariable model identified a number of independent predictors of receipt of IV antibiotics (Table 3). For example, personal care home residence and receipt of an antibiotic within the previous seven days increased the probability approximately fivefold and twofold, respectively. Higher temperature and respiratory rate were independently associated with receipt of the agents of interest, as was a recent history of vomiting. In addition, subjects in the highest 
Table 2 Characteristics of 3512 outpatients with community-acquired pneumonia: comparison by emergency department treatment

\begin{tabular}{|c|c|c|c|c|c|}
\hline \multirow[b]{2}{*}{ Patient variables } & \multicolumn{2}{|c|}{$\begin{array}{c}\text { No/oral antibiotic } \\
(\mathrm{N}=3339)\end{array}$} & \multicolumn{2}{|c|}{$\begin{array}{l}\text { IV antibiotic } \\
(\mathrm{N}=\mid 73)\end{array}$} & \multirow[b]{2}{*}{ P-value } \\
\hline & $\mathbf{N}$ & $\%$ & $\mathbf{N}$ & $\%$ & \\
\hline Age in years (median) & 47.7 & & 45.4 & & 0.31 \\
\hline Female gender & 1534 & 45.9 & 71 & 41.0 & 0.21 \\
\hline Personal care home residence & 74 & 2.2 & 19 & 11.0 & $<0.0001$ \\
\hline Antibiotic use in last 7 days & 588 & 17.6 & 40 & 23.1 & 0.07 \\
\hline Sought care for CAP last 7 days & 572 & 17.1 & 34 & 19.7 & 0.39 \\
\hline Nausea & 407 & 12.2 & 26 & 15.0 & 0.27 \\
\hline Vomiting & 381 & 11.4 & 37 & 21.4 & $<0.0001$ \\
\hline Temperature ${ }^{\circ} \mathrm{C}$ (median) & 37.1 & & 37.9 & & $<0.0001$ \\
\hline Heart rate - beats/minute (median) & 93.0 & & 101.0 & & $<0.0001$ \\
\hline Respirations per minute (median) & 20.0 & & 24.0 & & $<0.0001$ \\
\hline Diastolic BP (mmHg) (median) & 78.0 & & 72.0 & & $<0.0001$ \\
\hline Systolic BP (mmHg) (median) & 129.0 & & 125.0 & & $<0.05$ \\
\hline Risk Class & & & & & $<0.0001$ \\
\hline I & 665 & 19.9 & 20 & 11.6 & \\
\hline II & 1833 & 54.9 & 90 & 52.0 & \\
\hline III & 473 & 14.2 & 28 & 16.2 & \\
\hline IV & 320 & 9.6 & 25 & 14.5 & \\
\hline V & 48 & 1.4 & 10 & 5.8 & \\
\hline Triage Score & & & & & $\mathrm{P}<0.0005^{\mathrm{a}}$ \\
\hline 1 & I & 0.03 & 1 & 0.6 & \\
\hline 2 & 142 & 4.3 & 14 & 8.1 & \\
\hline 3 & 1529 & 45.8 & 81 & 46.8 & \\
\hline 4 & 1290 & 38.6 & 41 & 23.7 & \\
\hline 5 & 66 & 2.0 & 4 & 2.3 & \\
\hline Not documented & 311 & 9.3 & 32 & 18.5 & \\
\hline Time of day & & & & & $0.32^{b}$ \\
\hline Day (08:00-16:00 hours) & 1232 & 36.9 & 71 & 41.0 & \\
\hline Evening ( $16: 0 \mathrm{I}-24: 00$ hours) & 1429 & 42.8 & 65 & 37.6 & \\
\hline Night (00:0I-07:59 hours) & 472 & 14.1 & 28 & 16.2 & \\
\hline Not documented & 206 & 6.2 & 9 & 5.2 & \\
\hline
\end{tabular}

a Excludes those "not documented" and collapsed categories of triage scores into 3 severity levels (high; triage $=1$ or 2 , moderate; triage $=3$, low; triage $=4$ or 5 ).

b Excludes those "not documented".

Abbreviations: CAP, community-acquired pneumonia; IV, intravenous.

severity and risk classes were significantly more likely to receive IV antibiotics compared with those in the lowest severity and risk classes. Variables unrelated to patient demographics and severity of illness were also associated with receipt of IV antibiotics. For example, subjects presenting to EDs in the second year of the study had a decreased odds of receipt of IV treatment, while site of care continued to predict such treatment despite controlling for patient demographics and severity indicators. Odds of receipt of IV antibiotics at the site with the highest use was 14.2 times that at the site with the lowest use of IV antibiotics for CAP patients.

\section{Discussion}

In the current study, only a small proportion (approximately $5 \%$ ) of patients with CAP presenting to EDs and treated on an ambulatory basis received treatment with IV antibiotics. Antibiotic agents received by orally-treated subjects were primarily those recommended for outpatients in published treatment guidelines (eg, erythromycin, clarithromycin, or azithromycin) (Mandell et al 2000). In contrast, IV-treated subjects received agents consistent with recommendations for inpatients; eg, cefuroxime plus a macrolide (erythromycin, clarithromycin, or azithromycin) and levofloxacin alone (Mandell et al 2000).

In addition to site of care, multivariable analysis identified a number of independent predictors of receipt of IV antibiotics. The positive association of patient variables, such as inability to tolerate oral medications (eg, history of vomiting) and higher temperature and respiratory rate, with receipt of IV antibiotics suggests such treatment was being reserved for more acutely ill outpatients. The positive 
Table 3 Predictors of receipt of IV antibiotic in the emergency department: results of multivariable analysis

\begin{tabular}{|c|c|c|c|}
\hline \multicolumn{2}{|c|}{ Patient variable } & Odds ratio & $95 \% \mathrm{Cl}$ \\
\hline \multicolumn{2}{|c|}{ Study year (second) } & 0.37 & $0.26-0.52$ \\
\hline \multicolumn{2}{|c|}{ Temperature (units $=1{ }^{\circ} \mathrm{C}$ ) } & 1.68 & $1.43-1.96$ \\
\hline \multicolumn{2}{|c|}{ Respirations per minute (units $=5$ ) } & 1.23 & $1.06-1.43$ \\
\hline \multicolumn{2}{|c|}{ Vomiting } & 1.61 & $1.07-2.45$ \\
\hline \multicolumn{2}{|c|}{ Residence (Personal care home) } & 5.30 & $2.7 I-10.34$ \\
\hline \multicolumn{2}{|c|}{ Prior antibiotic treatment } & 1.99 & $1.33-2.98$ \\
\hline \multirow[t]{7}{*}{ Site: } & A & $|4.2|$ & $6.16-32.82$ \\
\hline & B & 7.93 & $3.46-18.19$ \\
\hline & $\mathrm{C}$ & 3.87 & $1.56-9.58$ \\
\hline & $\mathrm{D}$ & 2.27 & $0.88-5.84$ \\
\hline & $\mathrm{E}$ & 1.85 & $0.56-6.15$ \\
\hline & $\mathrm{F}$ & 1.56 & $0.59-4.09$ \\
\hline & $\mathrm{G}$ & 1.00 & referent \\
\hline \multirow[t]{3}{*}{ Severity: } & High (triage $=1$ or 2 ) & 3.15 & $1.55-6.38$ \\
\hline & Moderate (triage $=3$ ) & 1.29 & $0.88-1.90$ \\
\hline & Low (triage $=4$ or 5$)$ & 1.00 & referent \\
\hline \multirow[t]{5}{*}{ Risk class: } & $\mathrm{V}$ & 3.50 & $1.26-9.72$ \\
\hline & IV & 1.56 & $0.77-3.15$ \\
\hline & III & 1.53 & $0.8 I-2.9 \mid$ \\
\hline & II & 1.12 & $0.66-1.88$ \\
\hline & I & 1.00 & referent \\
\hline
\end{tabular}

Abbreviation: $\mathrm{Cl}$, confidence interval.

associations between more comprehensive measures of severity of illness, such as the risk class and triage score, with receipt of IV antibiotics was not unexpected. Similarly, the increased likelihood of receipt of IV treatment among personal care home residents suggests conservative care in light of the possible ramifications of inadequate treatment among this patient population.

Modest variations in the use of IV antibiotics among outpatients were evident among the seven sites. The highest proportion of outpatients receiving IV agents (10.6\%) was observed at site A. The lack of available inpatient beds at site A (free-standing ED) may have been a factor in the decision to prescribe IV antibiotics for patients who might otherwise have been admitted. However, the seven EDs studied are part of a regional healthcare system, and beds at all six hospitals are available to physicians at site A. Indeed, approximately $20 \%$ of such subjects presenting to site A were admitted to one of the other acute care hospitals. Site A evidenced the lowest hospitalization rate of all sites, although this may be attributed to differences in case mix rather than a greater propensity to prescribe IV agents. The higher use of IV antibiotics at site A remained, even when severity of illness was controlled for in the multivariable analysis. However, it appears the nature of site A (lack of readily available inpatient beds) may be an important consideration, given that physicians treating subjects at both sites A and B were significantly more likely to prescribe IV antibiotics to subjects at site A. Further, the heterogeneity in the types of centers sampled (eg, free-standing ED, vs attached to an acute care hospital) allowed for the determination of a range of IV antibiotic use which might be expected in the treatment of CAP.

The decreased use of IV antibiotics during the second year of data collection highlights a limitation of this study. Collection of this data coincided with implementation at all study sites of the critical pathway to guide clinicians in the management of both inpatients and outpatients with CAP This included quarterly performance reviews, which may have resulted in the decreased use of IV antibiotics as the study progressed. Inclusion of the study-year variable in the multivariable analysis controlled for this environmental factor, however, such intervention limits the generalizability of our findings. Specifically, receipt of IV antibiotics among outpatients with CAP in EDs may be more widespread than reported here.

Not all subjects had radiographic confirmed pneumonia. Rather, these data reflect physicians' practice patterns in treating suspected or presumed pneumonia. Limiting the analysis to subjects with radiographic confirmed pneumonia did not change study conclusions (data not shown). In addition, it is unclear, from these data, whether the decision regarding site of care (hospital vs community) preceded the decision to prescribe IV antibiotics or vice versa. If the decision to treat a subject as an outpatient preceded the prescribing decision, IV antibiotics may be considered part of an efficacious outpatient regimen. Alternately, IV antibiotics may have been employed as a means to stabilize and/or further assess "borderline" cases prior to making a decision regarding hospitalization. Both of these approaches may be beneficial: the former in providing improved treatment outcomes and preventing subsequent hospitalization, the latter in preventing unnecessary initial hospitalizations. Further study of both of these approaches to treatment would assist in determining the efficacy and efficiency of employing IV antibiotics for CAP in the ED.

Finally, this study did not include a comprehensive follow-up of outpatients. Differences in outcome between the different routes of administration and study sites and the effect of decreased use of IV agents in the second year of the study are unknown. Thus, we cannot comment upon the appropriateness of IV antibiotic use. Judicious use of IV antibiotics for treatment of CAP in EDs may prevent costly hospitalization, as noted above; however, further study 
is required to determine optimal use of this therapeutic alternative.

In summary, only a small proportion of outpatients received IV antibiotics for treatment of CAP in EDs, although the extent of such practice differed between hospital sites. A number of severity-related variables were associated with an increased probability of receipt of IV agents, however, differences between hospital sites persisted despite controlling for differences in case mix. While an initial dose of IV antibiotics in the ED has been suggested as part of an outpatient management strategy (Moran 2001), further study is required to determine the extent to which such use may prevent unnecessary hospitalization, and improve outcomes.

\section{Acknowledgments}

We thank the Pneumonia Pathway research nurses: JoAnne de Jager, Linda Gardner, Lynne Korobanik, Tammy Pfeiffer, Cynthia Proskow, Sue Marshall, Nancy Baker, Nan Horne, and Fredrika Herbert for their assistance. In addition, we are grateful to the members of the EPICORE data management center at the University of Alberta Hospital. Financial support for this study included an establishment grant from Alberta Heritage Foundation for Medical Research and grants in aid from Capital Health, Abbott Canada, Pfizer Canada, and Janssen-Ortho Canada.

\section{Conflict of interest}

TJM has been the recipient of grants from Abbott Canada, Pfizer Canada, and Janssen-Ortho Canada. AGC has been the recipient of a grant from Janssen-Ortho Canada.

\section{References}

Bartlett JW, Dowell SF, Mandell LA, et al. 2000. Practice guidelines for the management of community-acquired pneumonia in adults. Clin Infect Dis, 3:347-82.

Battleman DS, Callahan M, Thaler HT. 2002. Rapid antibiotic delivery and appropriate antibiotic selection reduce length of hospital stay of patients with community-acquired pneumonia: link between quality of care and resource utilization. Arch Intern Med, 162:682-8.

Fantin B, Aubert JP, Unger P, et al. 2001. Clinical evaluation of the management of community-acquired pneumonia by general practitioners in France. Chest, 120:185-92.

Fine MJ, Auble TE, Yealy DM, et al. 1997. A prediction rule to identify low-risk patients with community-acquired pneumonia. $N$ Engl J Med, 336:243-50.

Gilbert K, Gleason PP, Singer DE, et al. 1998. Variations in antimicrobial use and cost in more than 2,000 patients with community-acquired pneumonia. Am J Med, 104:17-27.

Gleason PP, Kapoor WN, Stone RA, et al. 1997. Medical outcomes and antimicrobial costs with the use of the American Thoracic Society Guidelines for outpatients with community-acquired pneumonia. JAMA, 278:32-9.

Jin Y, Marrie TJ, Carriere KC, et al. 2003. Variation in management of community-acquired pneumonia requiring admission to Alberta, Canada hospitals. Epidemiol Infect, 130:41-51.

Mandell LA, Marrie TJ, Grossman RF, et al. 2000. Canadian guidelines for the initial management of community-acquired pneumonia: an evidence-based update by the Canadian Infectious Diseases Society and the Canadian Thoracic Society. Clin Infect Dis, 31:383-421.

Marrie TJ. 1998. Community-acquired pneumonia: epidemiology, etiology, treatment. Infect Dis Clin North Am, 12:723-40.

Marrie TJ, Lau CY, Wheeler SL, et al. 2000. A controlled trial of a critical pathway for treatment of community-acquired pneumonia. CAPITAL Study Investigators. Community-Acquired Pneumonia Intervention Trial Assessing Levofloxacin. JAMA, 283:749-55.

Menendez R, Ferrando D, Valles JM, et al. 2001. Initial risk class and length of hospital stay in community-acquired pneumonia. Eur Respir $J, 18: 151-6$.

Moran GJ. 2001. New directions in antiinfective therapy for communityacquired pneumonia in the emergency department. Pharmacotherapy, 21:95S-99S. 